\title{
Following the standard form: Effects of equation format on algebraic modeling
}

\author{
Kristie J. Fisher • Katja Borchert • Miriam Bassok
}

Published online: 6 November 2010

(C) Psychonomic Society, Inc. 2010

\begin{abstract}
When people construct algebraic equations to represent quantitative relations, they often reverse the roles of the variables $(6 S=P$ instead of $6 P=S)$. Results from three experiments show that a major reason for such reversal errors is people's adherence to, and interpretation of, the Standard Form of algebraic models. College students constructed, selected, and interpreted algebraic models that either had the standard multiplication format (MF: $a x=y$ ), or the mathematically equivalent division format (DF: $y / a=x$ ). A large minority of participants constructed reversed MF models, whereas most participants who were instructed to use DF equations constructed correct models (Experiment 1). Most participants understood that MF and DF models of the same relation should be mathematically equivalent (Experiment 2), but they drew a conceptual distinction between these models (Experiment 3). The authors discuss the impact of notational conventions on people's interpretation and use of representational tools.
\end{abstract}

The experiments reported here formed the basis of Kristie Fisher's master's thesis (Experiment 1) and Katja Borchert's dissertation (Experiments 2 and a variant of Experiment 3). Both sets of studies were conducted at the University of Washington under the supervision of Miriam Bassok. The studies were presented, respectively, at the 48th and 41st annual meetings of the Psychonomic Society in Long Beach, CA, November 2007, and New Orleans LA, November 2000. Parts of this research were supported by the Royalty Research Fund (Grant 65-3610) to Miriam Bassok. We thank undergraduate research assistants Autumn Metzger, Marie Ng, Alex Schlee, Jeanne Sprague, Carly Thurston, and Greg Walsten for their help with data collection and coding.

K. J. Fisher $\cdot$ K. Borchert $\cdot$ M. Bassok $(\bowtie)$

Department of Psychology, University of Washington,

Box 351525, Seattle, WA 98195, USA

e-mail: mbassok@u.washington.edu
Keywords Algebra $\cdot$ External representation $\cdot$ Format . Mathematical reasoning

\section{Introduction}

Algebraic equations are often used as models of various quantitative relations. For example, the equation $y=a+b$ $(x-1)$ can represent the cost $(y)$ of parking several hours $(x)$ in a garage, which is charging a certain fee $(a)$ for the first hour of parking and a different fee $(b)$ for every additional hour of parking. Such algebraic models can help people reason about a variety of problem situations, if only because they provide concise representations of how the relevant variables relate to one another (Koedinger, Alibali, \& Nathan, 2008; Mochon \& Sloman, 2004; Sherin, 2001; Waldmann, 2007). Of course, the usefulness of algebraic models for reasoning and problem solving is limited to the cases in which the equations people construct properly correspond to the quantitative relations they attempt to model. Unfortunately, even at the college level, many students fail to construct correct algebraic models of quantitative relations that are quite straightforward.

The most striking example of erroneous algebraic modeling in college students comes from research on the infamous "Students and Professors" problem. About 30 years ago, Clement, Lochhead, and Soloway (1979) asked engineering majors to construct an algebraic equation to represent the statement, "At a certain university, there are six times as many students as professors," using $S$ to represent the number students and $P$ to represent the number of professors. Many of the participants in that study $(37 \%)$ failed to construct the correct algebraic equation $(6 P=S)$, and their most common error was a reversal of the two variables $(6 S=P)$. Over the years, these 
and other researchers have reported similar findings, with error rates ranging between 40 and 60\% (Bernardo \& Okagaki, 1994; Clement, 1982; Clement, Lochhead, \& Monk, 1981; Fisher, 1988; Graf, Bassok, Hunt, \& Minstrell, 2004; Kaput \& Sims-Knight, 1983; Martin \& Bassok, 2005; Rosnick, 1981; Rosnick \& Clement, 1980; Wollman, 1983).

The dominant explanation for the fact that college students commit reversal errors is their poor understanding of algebra, although people who commit such errors may hold distinct algebraic misconceptions (e.g., Clement, Lochhead, \& Monk, 1981; Cohen \& Kanim, 2005; Herscovics, 1989; Kieran, 1990, 1992; Lochhead \& Mestre, 1988; MacGregor \& Stacey, 1993; Rosnick \& Clement, 1980). For example, Clement et al. (1981) found that some college students did not understand that the letters $S$ and $P$ stand for variables (i.e., that $S$ denotes the number of students and $P$ denotes the number of professors). Instead, they interpreted these letters as unit labels ( $S$ denotes Students and $P$ denotes Professors). These students did not construct algebraic models, but rather, constructed unitconversion expressions, such as $10 \mathrm{~mm}=1 \mathrm{~cm}$, to represent their correct understanding that there are six students for every professor (Kieran, 1990; Kuchemann, 1981). Other college students in the Clement et al. study committed reversal errors because they translated the words of the relational statement into symbols in the order in which the words appeared in the statement (e.g., translating " 6 times as many students" into $6 S$, "as" into the equal sign $=$, and "professors" into $P$ ). Unlike students who failed to understand that, in algebra, letters denote variables, students who translated words into symbols believed that they actually constructed algebraic models.

Direct translation of verbal statements into algebraic equations (or "syntactic" translation; Herscovics, 1989) is a heuristic method that allows students to achieve a solution without engaging in the more effortful process of mathematical modeling, which would require thinking about the relative sizes of the compared sets and about the appropriate algebraic representation of the relational statement (e.g., Hinsley, Hayes, \& Simon, 1977). Students tend to rely on this heuristic because, for many relational statements (e.g., "The number of students is six times the number of professors"), the direct translation method actually leads to correct solutions (e.g., Graf et al., 2004). Because direct translation saves cognitive effort, even people who have good understanding of algebraic modeling may use it at times. Nonetheless, direct-translation solutions are much more common in students who have a relatively poor understanding of the target mathematical domain (Hinsley et al., 1977; Mayer \& Hegarty, 1996).

Irrespective of the specific reason that leads students to commit reversal errors, such modeling errors are much more common in algebra than in numerical problem solving
(Bernardo \& Okagaki, 1994, Martin \& Bassok, 2005; Wollman, 1983), a domain for which students have developed strong modeling skills. For example, Martin and Bassok (2005) asked middle school, high school, and college students to either construct algebraic equations that represent relational statements, such as "There are 6 times as many nails as screws," or to solve numerical word problems with the same relational statements, such as "A certain factory produces 6 times as many nails as screws. Yesterday, it produced 3,450 nails. How many screws did it produce?" At every grade level, students committed significantly more reversal errors on the algebraic task than on the problem-solving task. Importantly, almost all college students solved the numerical word problems correctly, indicating that they understood the relational statements and were able to model them using their knowledge of arithmetic operations. Yet, a high proportion of college students from the same population committed reversal errors on the mathematically isomorphic algebraic equation-construction task, as documented in previous studies.

The discrepancy between students' modeling success on the numerical problem-solving and the algebraic equation construction tasks described above suggests that, even at the college level, many students fail to notice, or fail to understand, the correspondence between algebraic models and arithmetic computations that are involved in the solution of numerical word problems. This conclusion would be consistent with a large body of evidence about the difficulties that beginning algebra students have in relating algebra to arithmetic (e.g. Herscovics, 1989; Herscovics \& Linchevski, 1994; Kieran, 1990, 1992; Knuth, Alibali, McNeil, Weinberg, \& Stevens, 2005; McNeil \& Alibali, 2000; Sfard, 1991; Stacey \& MacGregor, 1999). According to Kieran (1990), many of these difficulties stem from inconsistent instructional practices, which may lead students to infer that algebra and arithmetic are governed by different sets of rules. One of the illustrations she presents is the different use of number and letter combinations in arithmetic (e.g., $12 \mathrm{~m}$ can mean 12 meters) and in algebra (e.g., $12 \mathrm{~m}$ can mean 12 times some unspecified number of meters), which could explain the variable-label misconception mentioned earlier.

The studies we report here were motivated by Kieran's (1990) conjectures about the impact of inconsistent notational conventions on students' difficulties in understanding algebra and its relation to numerical problem solving. We have identified a notational convention that pertains to the format of algebraic models, the Standard Form, and we examined whether this format convention may account for the high proportions of reversal errors in college students who, one would assume, have a better understanding of algebraic modeling than do beginning algebra students. The 
standard form of algebraic models has a multiplication format (e.g., $a x=y$ ). ${ }^{1}$ It is presented to students as being the "best form to represent a situation" (e.g., Larson, Kanold, \& Stiff, 1993, p. 264), and students are explicitly instructed that all rational expressions of variables (e.g., $x / a=y$ ) should be "undone" (e.g., Rubenstein et al., 2002, p. 315).

Previous work in our laboratory revealed that, as one would expect, most college students adhere quite strictly to this format convention when they construct algebraic models of relational statements (Borchert, 2003). We reasoned that because the standard form of algebraic models is similar to the linear, left-to-right form of verbal relational statements, it encourages reliance on the directtranslation shortcut heuristic. Note that, unlike in the case of numerical problem solving, people who construct algebraic models do not have access to numerical values (e.g., "There are 3,450 nails") that could help them avoid or correct such translation errors. This difference between algebraic modeling and numerical problem solving could explain why college students commit translation errors in algebra even though they correctly solve mathematically isomorphic word problems. Furthermore, it is quite possible that, although college students tend to engage in direct translation when they construct standard-form models, they do have sufficient understanding of algebra to construct correct algebraic models in a format that does not afford reliance on direct translation.

Our first experiment was designed to examine this possibility. Specifically, we asked one group of college students to construct algebraic models that had the standard, multiplication format (MF: $a x=y$ ), and asked another group of students from the same population to construct division-format models (DF: $x / a=y$ ). Because DF models do not afford reliance on the direct translation heuristic, students who have good understanding of algebraic modeling should perform better when they construct DF than when they construct MF equations. Hence, we expected to find better modeling performance in the DF than in the MF condition.

In addition to a possible detrimental influence of the similarity between the formats of algebraic models and verbal statements, we suspected that the standard form of

\footnotetext{
${ }^{1}$ Note that in textbooks the "formal" version of the standard form is usually written with the addition of a constant and with the variables on the same side of the equal sign, $A x+B y=C$. The literature on reversal errors presents equations in which the single variable always appears on the right side of the equal sign $(6 S=P)$ and does not distinguish between the left-right positions of the single variable $(S=6 P$ vs. $6 P=S$, respectively). We followed this convention in Experiment 1, such that in all the example equations we provided the single variable was on the right side of the equal sign (e.g., $6 P=S$ ). Following a reviewer's question, in Experiments 2 and 3 we examined if the leftright orientation interacts with participants' performance and did not find any evidence of such an interaction.
}

algebraic models could be understood as a potentially meaningful "discontinuity" between algebra and arithmetic, of the sort discussed by Kieran (1990). If so, it may hinder students' ability to understand the conceptual relation between modeling in algebra and in numerical problem solving. In the context of numerical problem solving, mathematical modeling entails choosing an arithmetic operation that corresponds to the situation described in the problem text. College students have extensive experience with this modeling task, and are highly fluent in selecting arithmetic operations that are structurally analogous to the quantitative relations in word problems (e.g., Bassok, Chase, \& Martin, 1998; Bassok, Wu, and Olseth, 1995; Dixon, 2005; Dixon, Deets \& Bangert, 2001; Martin \& Bassok, 2005). When students transition to algebra and learn that algebraic models have a standard (multiplication) form, some of them may be unsure whether and, if so, how algebraic modeling involves selecting an appropriate arithmetic operation. As a result, they may refrain from engaging in algebraic modeling when constructing equations and, instead, resort to reliance on the direct translation heuristic.

If, indeed, people engage in direct translation because they do not understand the relation between standard-form algebraic models and the models used to solve numerical word problems, they may fail to realize the mathematical and functional equivalence of standard form (MF) and nonstandard form (DF) algebraic models. To test this possibility, in Experiment 2 we examined whether college students realize that MF and DF models of the same relational statement have to be mathematically equivalent, and in Experiment 3, we examined whether college students come up with similar modeling interpretations of mathematically isomorphic MF and DF equations. That is, Experiments 2 and 3 were designed to test the possibility that, in addition to eliciting reliance on the direct translation heuristic (Experiment 1), the standard form of algebraic models also leads people to draw a conceptual distinction between MF and DF representations of relational statements.

\section{Experiment 1}

In Experiment 1, we examined the extent to which the standard form of algebraic models elicits reliance on the direct-translation heuristic because of its similarity to the left-to-right word order in verbal statements and, therefore, contributes to the high proportion of reversal errors in college students. As we mentioned above, we asked one group of college students to represent relational statements of the form, "There are $\mathrm{N}$ times as many $\mathrm{X}$ as $\mathrm{Y}$ " using the standard form multiplication-format equations (MF), and we asked another group of college students to 
represent these same statements using division-format equations (DF). Unlike the standard-form MF equations, DF equations do not afford reliance on the direct translation heuristic for this type of relational statement. Hence, to the extent that college students do understand algebra and can construct correct algebraic models, they should be more likely to construct correct models in the DF condition, when they must engage in algebraic modeling, than in the MF condition, when they can translate words into equations.

In addition to comparing the proportions of correct algebraic models participants constructed in the two format conditions, we recorded the time they took to read the relational statements they were asked to model. Under the assumption that this reading time includes planning of the algebraic equation participants constructed, we expected reading times corresponding to correct solutions, which necessitate modeling, to be longer than reading times corresponding to reversal errors, which are likely to reflect reliance on the non-modeling direct translation method. Also, because the participants in our study had significantly more experience in dealing with standard (MF) than with non-standard (DF) algebraic models, we expected that they would take more time to plan the construction of the DF than of the MF equations.

\section{Method}

\section{Participants}

The participants were 121 introductory psychology student volunteers (46 male, 75 female) from the University of Washington $^{2}$. Almost half (48\%) were in their first year at the university, and the vast majority $(88 \%)$ of participants indicated that they had completed calculus. Fifty-five percent stated that they were majoring in or planned to major in the sciences or engineering. They received course extra credit for their participation.

\section{Materials}

We constructed eight multiplicative-comparison statements, similar to those used by Martin and Bassok (2005). All statements were of the form, "There are $\mathrm{N}$ times as many $\mathrm{X}$

\footnotetext{
${ }^{2}$ We did not collect any further demographic information. According to the statistics released by the University of Washington regarding recent graduating classes, approximately $54 \%$ of the students are White, $3 \%$ are Black, $25 \%$ are Asian/Pacific Islander, $4 \%$ are Hispanic, $1 \%$ are Native American, and $11 \%$ are of other or unknown ethnicity. Admissions data from 2008-2009 indicate that the middle $50 \%$ of students had math SAT scores between 550 and 680 and critical reading scores between 520 and 640. Fifty-two percent of admitted students were female.
}

as Y" (e.g., "There are 6 times as many nails as screws") and contained sets of distinct objects. Half of the paired object sets belonged to the same taxonomic category (nails and screws; cups and mugs; skirts and dresses; muffins and brownies), and the other half paired functionally related object sets (roses and vases; cookies and jars; apples and baskets; students and professors) ${ }^{3}$. Each of the four categorical and thematic statements had a different numerical constant: 3, 4, 5, or 6. We used E-Prime 1.1 [SP3] software to create a computerized version of the equation construction task, which was displayed to participants on 15-inch Dell computer monitors.

\section{Procedure}

Participants performed the task in a computer lab in groups of one to 12 . They first read on-screen text informing them that algebraic equations use variables to represent quantities, and that these variables are represented by letters. They also read that algebraic equations could involve both variables and specific numbers, and that the variables and the numbers are related by arithmetic operations. Participants then received four examples, one for each of the four arithmetic operations: addition $(X+8=Y)$, subtraction $(X-$ $8=Y)$, multiplication $(X * 8=Y)$, and division $(X / 8=Y)$. The order in which they saw these examples was counterbalanced across participants. The purpose of this introductory explanation and the examples was to disambiguate the subsequent instructions to the target task, in which participants were asked to construct either an MF or a DF equation.

After they finished reading this introductory text, participants were informed that they would be asked to construct equations in different formats to represent various statements. Participants were also informed that, before each statement appeared, they would be told whether they should construct an addition, subtraction, multiplication, or division equation. They were then asked to construct an addition equation to represent the statement "There are 5 more books than magazines," and a subtraction equation to represent the statement "There are 3 more boys than girls." The order of these two equation-construction tasks, which served as practice trials, was counterbalanced across parti cipants. No feedback was provided. After these practice trials, participants were either asked to construct an MF equation $(N=62)$ or a DF equation $(N=59)$. The relational statement seen by each participant was randomly selected from the set of eight relational statements created for the experiment.

\footnotetext{
${ }^{3}$ We used both types of object pairs to control for the possibility that they may interact with participants' performance on the MF and the DF equations. Consistent with the findings of Martin and Bassok (2005) that pertain to college students, we did not find any such effects.
} 
To initiate each equation-construction task, participants pressed the space bar. A relational statement then appeared on the screen, and participants were instructed to press the space bar when they had finished reading the statement. Reading time (in seconds) was recorded between these two space bar presses. After the second key press, the statement disappeared and was replaced by instructions to construct an equation to represent the statement that the participant had just seen using the provided variables. The variables were the initial letters of the paired object sets (e.g., $N$ and $S$ for the sets nails and screws, respectively). Participants typed their answer into an input box. Most participants finished the task in less than 10 minutes.

Results

\section{Correct performance}

We classified answers as "Correct", "Reversal" errors, and "Other" errors (e.g., Clement, Lochhead, \& Monk, 1981). Reversal errors inverted the variable roles from their position in the correct MF equations (e.g., $6 S=P$ for the correct equation $6 P=S$ ), or in the corresponding $\mathrm{DF}$ equations (e.g., $P / 6=\mathrm{S}$ for the correct equation $S / 6=P$ ). Other errors were expressions that did not include an equal sign (e.g., $6 S / P$ ), inequalities (e.g., $6 S>P$ ), or other nonreversal equations (e.g., $6 / P=S$ ).

Two participants in the DF condition and three in the MF condition constructed equations using the variables " $\mathrm{X}$ " and " $Y$ " instead of using the variables we provided. These five responses were impossible to code as correct or incorrect according to our definitions above and thus were excluded from the analyses. Moreover, seven of the participants in the DF condition ignored the instructions to construct a division equation and, instead, constructed MF equations. Six of them constructed a correct MF equation and one constructed a reversed MF equation. Interestingly, only one of the participants in the MF condition constructed a DF equation, which contained a non-reversal error. This pattern of non-compliant responses in the DF and MF conditions is consistent with students' adherence to the standard equation form of algebraic models. We excluded these eight noncompliant responses from our analysis, although retaining them does not change the pattern of the reported results. The frequencies of Correct, Reversal, and Other valid responses in the MF $(N=58)$ and $\mathrm{DF}(N=50)$ conditions appear in Table 1.

As can be seen in Table 1, the response distributions in the two format conditions were significantly different, $\chi^{2}$ $(2, N=108)=7.81, p=0.02$. Consistent with our prediction that the standard form may evoke non-modeling translation responses even in students who can engage in correct algebraic modeling, the overall percentage of correct
Table 1 Percent Correct, reversal error and other error algebraic models constructed in the multiplication format (MF) and division format (DF) equation conditions in Experiment 1

\begin{tabular}{llll}
\hline Format & Correct model & Reversal error & Other error \\
\hline MF $(N=58)$ & $64 \%$ & $29 \%$ & $7 \%$ \\
DF $(N=50)$ & $84 \%$ & $8 \%$ & $8 \%$ \\
\hline
\end{tabular}

algebraic models in the DF condition was quite high $(84 \%)$, and it was significantly higher than in the MF condition $(64 \%)$. In a complementary way, the frequency of responses categorized as Reversal errors was significantly higher in the MF condition (29\%) than in the DF condition (8\%). Given that division-format equations do not afford direct-translation solutions, the fact that some participants constructed reversal-error DF equations suggests that they first used direct translation to construct a standard-form MF equation and, subsequently, transformed it into a DF equation. Finally, the frequencies of Other-error responses were similarly low in both the MF (7\%) and the DF (8\%) conditions.

\section{Reading time}

Table 2 presents the means and the standard deviations of the time participants took to read the relational statements ${ }^{4}$. The results are consistent with our assertion that direct translation of words into symbols, which for the statements we used here results in reversal errors, circumvents the more effortful process of algebraic modeling. Specifically, participants who constructed correct equations took significantly longer to plan the equations they constructed $(M=$ $12.51, S D=10.63)$ than did those who constructed reversed equations $(M=8.65, S D=5.80), t(98)=2.58, p=0.01$. Moreover, consistent with their differential experience in dealing with $\mathrm{MF}$ and $\mathrm{DF}$ equations, participants took significantly longer to plan non-standard DF models $(M=$ $13.83, S D=12.28)$ than they took to plan the standard-form MF models $(M=9.50, S D=5.86), t(106)=3.10, p=0.003$. As can be seen in Table 2, this difference held true for all response categories (Correct, Reversal, and Other).

\section{Discussion}

College students in Experiment 1 exhibited a very different performance pattern when asked to construct standard form

\footnotetext{
${ }^{4}$ The $t$-test was conducted on the log-transformed data (Smith, 1976) because of large variance. Reported descriptive mean was transformed back from the mean of log seconds. Standard error was calculated by first defining the $95 \%$ confidence interval of the mean of the log score, converting back into seconds, and then dividing the difference between the upper and lower bounds by two.
} 
Table 2 Mean reading times (M), in seconds, and standard deviations (SD) of correct, reversal error and other error algebraic models constructed in the multiplication format (MF) and division format (DF) equation conditions in Experiment 1

\begin{tabular}{|c|c|c|c|c|c|c|c|c|}
\hline \multirow[t]{2}{*}{ Format } & \multicolumn{2}{|c|}{ Correct model } & \multicolumn{2}{|c|}{ Reversal error } & \multicolumn{2}{|c|}{ Other error } & \multicolumn{2}{|l|}{ Total } \\
\hline & M & SD & M & SD & M & SD & M & SD \\
\hline $\operatorname{MF}(N=58)$ & 10.28 & 6.01 & 7.95 & 5.80 & 8.91 & 4.17 & 9.50 & 5.86 \\
\hline $\mathrm{DF}(N=50)$ & 14.47 & 13.22 & 11.60 & 5.49 & 9.30 & 2.23 & 13.83 & 12.28 \\
\hline Total & 12.51 & 10.63 & 8.65 & 5.80 & 9.11 & 3.10 & 11.50 & 9.60 \\
\hline
\end{tabular}

(MF) versus non-standard (DF) equations to represent identical relational statements. When asked to construct a standard-form MF equation, students' performance was consistent with previous studies that did not specify format requirements in the equation construction task instructions (Bernardo \& Okagaki, 1994; Clement, 1982; Clement et al., 1981; Fisher, 1988; Graf et al., 2004; Kaput \& Sims-Knight, 1983; Martin \& Bassok, 2005; Rosnick, 1981; Rosnick \& Clement, 1980; Wollman, 1983). Namely, just over $60 \%$ of participants in the MF condition constructed a correct algebraic model, and the vast majority of errors were reversal equations. In contrast, participants who were asked to construct a non-standard DF equation, constructed correct algebraic models at a frequency more similar to the performance levels observed when people are asked to solve isomorphic word problems (Martin \& Bassok, 2005).

The reading-time results support our entering conjecture that the standard form of algebraic models fosters reliance on the non-modeling direct-translation heuristic, and that college students take more time planning their non-standard (DF) than the standard (MF) modeling responses. It therefore appears that, when prevented from following the standard form, the participants in Experiment 1 were forced to stop and think about how to model the relational statement rather than just automatically implementing the direct translation heuristic. When they did, the vast majority of them had sufficient understanding of mathematical modeling to construct correct DF models of relational statements.

The results of Experiment 1 appear to contradict the common view that reversal errors reflect poor understanding of algebra. However, the high proportion of correct DF models in Experiment 1 does not, in itself, indicate that the participants in this study had good understanding of algebraic modeling. In particular, it is possible that they did not view the DF equations they constructed as being equivalent to what they believe to be algebraic models (i.e., MF models). Rather, they may have treated DF equations as representations of problem solving solutions. Experiments 2 and 3 were designed to test the possibility that college students draw a conceptual distinction between mathematically equivalent MF and DF representations of relational statements.

\section{Experiment 2}

The main question of interest in Experiment 2 was whether college students realize that MF and DF models of the same relational statement should be mathematically equivalent. To this end, we asked participants to select from an array of ten equations (MF, DF, and Foils) all the equations they considered to be correct representations of a given relational statement. The MF and DF equations in the array were mathematically isomorphic; half of the equations in each format were correct models of the relational statements, and the other half were reversal-error equations. Based on the results of Experiment 1, we expected that a large majority of the participants in Experiment 2 would select correct DF equations. We reasoned that, if college students who select correct DF equations realize that MF and DF models of the same statement are mathematically equivalent, they should be able to select correct MF equations. If, however, students draw a conceptual distinction between MF and DF representations of relational statements, they may select combinations of non-isomorphic correct DF and reversed MF models.

After they finished the equation-selection task, participants were asked to indicate which equation, of all the ones they deemed to be correct, they considered to be the "best" representation of the relational statement and to explain why they chose that particular equation. Given participants' experience with the standard form, we expected that, irrespective of their algebraic performance, most participants would prefer MF to DF equations. We examined how the participants justified their equations preferences, in the hope that these justifications would shed some light on their understanding of the standard-form convention.

\section{Method}

\section{Participants}

The participants were 94 introductory psychology students (29 male, 44 female, 21 did not indicate their gender) from the University of Washington (age: $M=18.61, S D=1.94$ ). No other demographic information was collected (see again note 2). Students received course extra credit for their participation. 


\section{Materials}

Relational statements We constructed four multiplicativecomparison statements ${ }^{5}$ (e.g., "There are 6 times as many nails as screws"), each with a different numerical constant $(4,5,6$, and 9). Two of the statements in each set paired objects that belonged to the same taxonomic category (e.g., peaches and apricots), and the other two statements paired functionally related objects (e.g., roses and vases; see again note 2.).

Equation set For each statement, we constructed a set of ten equations. We explain the composition of this equation set for the statement "There are 6 times as many nails as screws," with $N$ standing for the number of nails and $S$ standing for the number of screws. Each equation set included four MF equations, two correct $(6 S=N, N=6 S)$ and two reversed $(6 N=S, S=6 N)$; and four DF equations, two correct $(N / 6=S, S=N / 6)$ and two reversed $(\mathrm{S} / 6=N$, $N=\mathrm{S} / 6)$. It also included two incorrect foil equations, each with two operations: multiplication and addition $(6 N+S=$ total) and division and subtraction $(N=($ total $-S) / 6)$.

Questionnaires Each questionnaire consisted of a single page, with one of the four relational statements printed at the top. The relevant equation set was displayed in the cells of a $2 \times 5$ table that was printed below the statement. To control for order effects, the ten equations were presented in three different random orders. Participants were informed that the letters in the equations stand for the number of objects in the two sets mentioned in the statement (e.g., "In all equations, $N$ represents the "number of nails" and $S$ represents the "number of screws"). They were first instructed to read the statement and then circle all the equations that could represent the statement correctly. Next, participants were asked to choose one of the equations they selected that was, in their opinion, the best representation of the relational statement. Finally, participants were asked to explain their "best equation" choice.

\section{Procedure}

Participants were randomly assigned to receive one of four statements. The questionnaire was distributed to participants, along with questionnaires from other, unrelated studies, in a large classroom setting.

\footnotetext{
${ }^{5}$ Participants in another condition received statements of the form "For every $N X$ s there is one $Y$," so that we could see whether the presence of the word 'times' influenced participants' likelihood to select MF equations. It did not, and so we only report findings for statements similar to those in Experiment 1.
}

Results

\section{Equation selection}

Only 26 participants (28\%) selected all four correct equations (the two MF and the two DF) and no incorrect equations. Despite this fact, on average participants chose almost four equations, irrespective of correctness $(M=3.79$, $S D=2.00$ ), suggesting wide variety in participants' equation selection in terms of equation format and equation correctness. Table 3 presents the distribution of participants who selected various combinations of correct and incorrect MF and DF models. For each format, we distinguished between participants who selected (1) only correct models, (2) only reversal models, (3) both correct and reversal models, or (4) none of the models in that format.

First, as can be seen in Table 3, a large majority of the participants in Experiment 2 (89\%) selected at least one DF equation, and the proportion of participants who selected at least one MF equation was only somewhat higher (96\%). In fact, $88 \%$ of the 94 participants in this experiment selected both MF and DF equations. These results indicate that, when DF equations are available to them, most college students accept DF equations as legitimate representations of relational statements.

We further examined whether the MF and DF equations participants selected were mathematically equivalent. We found that this was the case for 71 of the 83 participants (86\%) who selected both MF and DF equations. These 71 participants make up $75 \%$ of the total sample $(N=94)$. That is, although some college students in our sample did not select models of both formats (12\%), or selected nonisomorphic models $(13 \%)$, a large majority of the participants in Experiment 2 did understand that MF and DF algebraic models of the same relational statement have to be mathematically equivalent. Interestingly, contrary to what we expected, their understanding of the mathematical equivalence of MF and DF models did not prevent participants from selecting reversed MF equations. In fact, more than one third $(35 \%)$ of the 71 participants who selected mathematically isomorphic models of both formats selected reversed MF and DF models. Most likely, these participants first selected the reversed MF equation and then, to be consistent, selected a mathematically isomorphic (reversed) DF equation.

Thus, whereas we thought that the presence of correct DF equations in the array would lead participants to select correct rather than reversed MF equations, it was the presence of reversed MF equations in the array that led participants to select reversed rather than correct DF equations. As a result, the proportion of participants who selected only correct DF equations $(55 \%)$ was smaller than expected and was comparable to the proportion of 
Table 3 Frequency distribution of participants who selected various combinations of correct and reversal error multiplication format (MF) and division format (DF) equations in Experiment 2

\begin{tabular}{|c|c|c|c|c|c|c|}
\hline \multirow[t]{2}{*}{ Format } & & \multicolumn{5}{|c|}{ Division format (DF) } \\
\hline & & Correct & Reversal error & Correct and reversal & None & Total \\
\hline \multirow[t]{5}{*}{ Multiplication format (MF) } & Correct & 46 & 0 & 0 & 2 & 48 \\
\hline & Reversal error & 5 & 25 & 6 & 3 & 39 \\
\hline & Correct and reversal & 0 & 1 & 0 & 2 & 3 \\
\hline & None & 1 & 0 & 0 & 3 & 4 \\
\hline & Total & 52 & 26 & 6 & 10 & 94 \\
\hline
\end{tabular}

participants who selected only correct MF equations $(51 \%)$, and these proportions are composed primarily of the participants who selected only correct equations of both formats (see again Table 3). Note that in Experiment 1 , in which participants were asked to construct either DF or MF equations, the respective frequencies of correct algebraic models were much higher (84\% and $64 \%$, respectively, for the $\mathrm{DF}$ and $\mathrm{MF}$ equations). In the discussion section, we address the apparent discrepancy in students' algebraic competence in the construction and the selection tasks.

\section{Equation preference}

Not surprisingly, the vast majority of the participants preferred the familiar standard-form to non-standard form algebraic models. In response to the question which of the equations they selected was the best representation of the relational statement, $86 \%$ of the participants chose an MF equation and only $7 \%$ indicated their preference for a DF equation. The remaining participants $(6 \%)$, either chose one of the foil equations or wrote an equation not listed on the page.

Participants generated a variety of non-exclusive justifications to explain their equation preference. A high proportion of these justifications were verbal re-statements of the chosen equations (e.g., "Multiplying \# of screws by 6 would equal $N$ "), or meta-cognitive statements (e.g., "It's a guess"), which cannot shed any light on participants' beliefs about algebraic models. Nonetheless, a substantial proportion of the justifications $(40 \%)$ mentioned more experience with using the standard form MF equations (e.g., "One does not normally look for division with this sort of questions"), or the relative ease of using MF rather than DF equations ("I find it easier to deal with Multiplication than Division"). Interestingly, a small subset of the justifications $(8 \%)$ explicitly mentioned that MF equations afford reliance on the direct-translation strategy (e.g., "The equation explains the statement in the order in which the two variables were stated"), although some of the parti- cipants who gave these justifications actually selected correct equations.

\section{Discussion}

The results of Experiment 2 show that most college students accept DF equations as possible algebraic models, and that a significant proportion of them understand that MF and DF models of the same relational statement should be mathematically equivalent. Yet, contrary to what we predicted based on students' ability to construct correct DF equations (Experiment 1), this understanding did not help them notice the variable reversals in the MF equations. Instead, it led them to select incorrect DF equations that were isomorphic to the reversed MF models they could readily construct by translating words into symbols. Because constructing correct DF models takes more planning time than constructing reversed MF models (Experiment 1), in hindsight, it is not surprising that participants identified the reversed MF model faster than it would take them to check, independently, the correctness of the DF models in the array. Because they understood that the MF and the DF equations should be isomorphic, they transformed the reversed MF equation into an equivalent reversed DF equation.

The above account can explain the discrepancy between students' modeling success in Experiment 1, where only a small minority of participants $(8 \%)$ constructed reversed DF models, and in Experiment 2, where a substantial proportion of participants (28\%) selected reversed DF models as being correct. In Experiment 1, participants who were asked to construct a DF model could not engage in direct translation and, therefore had to engage in algebraic modeling. By contrast, the equation array in Experiment 2 contained MF equations that afford direct translation. Because the reversed MF model was readily available to them, participants apparently transformed it into the division format without devoting the time needed to construct, or interpret, a DF model. Note that the way participants were tested in Experiments 1 and 2, in a 
computer lab vs. in a large classroom, respectively, may have also contributed to the difference in the time and effort they devoted to the algebraic modeling task.

We also found that, as one would expect, the participants in Experiment 2 had a very strong preference for MF over DF models. Those of them who gave an informative explanation for their format preference mentioned their higher familiarity with the standard form, the relative ease of using MF equations, and even the one-to-one correspondence between the order of the words in relational statements and the order of symbols in MF models. Experiment 3 aimed to examine whether, in addition to their high familiarity with using the standard form, the fact that people's algebraic modeling experience was restricted to using the standard form has led them to infer that DF equations serve a different function than do standard-form models. Specifically, as we have suggested in the Introduction, the non-standard DF equations may remind people of the computations that are involved in solving numerical word problems. If so, people may infer that, whereas standard-form MF equations are algebraic models that represent relational statements, DF equations represent the computations involved in solving numerical word problems.

\section{Experiment 3}

Experiment 3 aimed to examine whether, despite their ability to construct correct DF models (Experiment 1), and their understanding that MF and DF representations of the same quantitative relation have to be mathematically equivalent (Experiment 2), college students interpret standard-form models as representations of relational statements, but interpret the non-standard division-form models as representations of problem-solving solutions. To test this possibility, we presented college students with either an MF equation or with a mathematically equivalent DF equation, and asked them to describe a relation between two "real world" quantities that could correspond to the mathematical relation depicted in the equation. We then examined whether participants came up with different modeling interpretations of the MF and DF equations. Specifically, building on the distinction made by Kieran (1990) and Sfard (1991), and the linguistic analysis discussed by MacGregor and Stacey (1993), we developed a categorization scheme that distinguished between Equivalence (E) interpretations that view algebraic equations as representing equivalence relations between variable quantities, and Computation (C) interpretations that view such equations as representing problem-solving solutions. We then examined whether standard-form equations (MF) evoke more equivalence interpretations (and fewer computation interpretations) than do DF equations.
We also examined whether participants' modeling interpretations were correct, expecting that DF equations would elicit more correct modeling interpretations than would MF equations. We made this prediction for two reasons. First, unlike MF equations, DF equations do not afford reliance on the mindless direct translation method. Second, we expected that DF equations would elicit more computational interpretations than would MF equations, and that such interpretations should benefit from college students' good understanding of mathematical modeling in the context of solving numerical word problems (e.g., Martin \& Bassok, 2005).

\section{Method}

\section{Participants}

The participants were 72 college students (26 male, 46 female) from the University of Washington enrolled in introductory psychology classes, with a mean age of $18.96(S D=1.35)$. Of the 72 participants, 59 indicated that they had either completed or were currently enrolled in calculus.

\section{Stimuli}

We constructed two sets of mathematically isomorphic MF and DF equations that contained a constant and the variables "X" and "Y," (e.g., $6 Y=X$ and $X / 6=Y$, respectively). In each set, there were 16 equations that differed in their constants $(6,7,8$, or 9$)$ and in whether the $\mathrm{X}$ and $\mathrm{Y}$ variables appeared on the left or on the right side of the equation sign (e.g., $X / 7=Y$ or $Y / 7=X$ ). Moreover, in half of the equations the single variable appeared on the left of the equal sign (e.g., $Y=7 X$ ), as is customary in algebra, and in the other half it appeared on the right of the equal sign (e.g., $7 X=Y$ ), as is customary in solutions of arithmetic problems. Interestingly, this difference in conventional order did not affect the nature of participants' modeling interpretations (see again note 1).

Each of the MF and DF equations was printed on a separate page together with a set of instructions, and each participant received one such page. The instructions asked the participants to think about a relation between two quantities that could correspond to the mathematical relation between the $\mathrm{X}$ and $\mathrm{Y}$ variables in the equation. Participants were further instructed that the variables should refer to sets of countable objects, such as cupcakes, coins or people (these object sets were given as examples), and were asked to define their variables by filling in the blanks in the following statements: "X represents the number of " and "Y represents the number of _." Next, participants were asked to write a simple English sentence that described the 
relation between the two variables they chose and corresponded to the equation.

\section{Procedure}

The equation-interpretation questionnaires were administered to participants in groups of 10-25. Participants were randomly assigned to one of the format conditions. Thirtyfive participants received MF questionnaires, and 37 received DF questionnaires. They were given informed consent and told to carefully read and follow the questionnaire instructions; most participants completed the task in less than 10 minutes.

\section{Results}

Here, we first report results showing that participants generated qualitatively different modeling interpretations for the MF and DF equations. Then, we report results pertaining to the correctness of these interpretations in the MF and DF conditions.

\section{Equation interpretations}

We classified the modeling interpretations as either Equivalence (E) or Computation (C). Interpretations coded as $\mathrm{E}$ were verbal statements that expressed a quantitative equivalence between two object sets. Our coding scheme focused on the presence or absence of key words and phrases. Statements coded as E typically consisted of a single sentence. These sentences contained phrases such as, "There are," "For every," or "Times as many," or expressions describing direct or inverse variation, such as, "As $\mathrm{X}$ increases, $\mathrm{Y}$ increases." Examples of $\mathrm{E}$ interpretations are: "For every seven athletes on an athletic team, there is one coach," or "There are $1 / 8$ as many spiders as there are spider legs." Interpretations coded as C were word-problem texts and/or explicit descriptions of calculations. These interpretations contained phrases such as, "divided by," "multiplied by," or "In order to calculate the number of," explicitly referenced quantities, or described actions that were analogous to mathematical operations, such as, "A pizza is sliced up into seven slices." Examples of responses in the $\mathrm{C}$ category are, "Each unit is worth $\$ 7$ in profit, and multiplying that by the units sold $(\mathrm{Y})$ yields the amount of profit (X)," or "With one coin, you can do the laundry 8 times, with 2 coins, you can do the laundry 16 times, etc."

There were a few cases where the words "is" or "are" could potentially be interpreted as either representing equivalence or a computation, such as in, "One insect has 6 feet. The number of their feet is six times the number of insects." In such cases, the categorization of $\mathrm{C}$ versus $\mathrm{E}$ was made based on the presence or absence of other phrases and the general structure of the interpretation statement. In the above example, the interpretation was coded as Computation because the participant first stated a specific quantity then used the phrase "number of" when describing the relation between the variables. Because this was more similar to the structure of a word problem than a singular equivalence statement, it was coded as C. Note that, if at all, this coding decision overestimates the number of computational interpretations for the MF statements. The first author $(\mathrm{KF})$ coded all the data, and an independent rater (AM) coded a random sample of $35 \%$ of the data. The two coders overlapped in $93 \%$ of the cases. Disagreements between the coders were resolved, and the remaining $65 \%$ of interpretations were then re-checked by $\mathrm{KF}$ to ensure consistency with decisions made about disputed cases. One participant was excluded from the analyses because the coders could not agree on the category of this participant's interpretation. The final dataset consisted of 71 responses, 34 from participants in the MF condition and 37 from the DF condition.

Using the above coding scheme, we found that $\mathrm{E}$ interpretations were significantly more common in the MF condition $(76 \%)$ than in the DF condition $(49 \%), \chi^{2}(1, N=$ $71)=5.82, p=0.02$. Thus, as we have suspected, a significant proportion of college students made a conceptual distinction between MF and DF models.

\section{Interpretation correctness}

As in the previous two experiments, we classified participants' responses as "Correct", "Reversal" errors, and "Other" incorrect or vaguely worded statements. Reversal errors were defined as statements that would be correct if the positions of the two quantities in the statement had been reversed. Table 4 presents illustrative examples of these three response categories and their relative frequencies in the MF and DF conditions.

As can be seen in Table 4, and consistent with our prediction, participants were much more likely to generate correct modeling interpretations for the DF equations (76\%) than for the MF equations (44\%). The complementary pattern held for the relative proportions of Reversal errors ( $16 \%$ and $44 \%$ for the DF and MF equations, respectively). The relative proportions of Other incorrect interpretations in the two format conditions were similarly low $(12 \%$ and $8 \%$, respectively). A homogeneity-of-proportions test revealed a significant difference between the distributions of correct and incorrect responses in the MF and DF conditions, $\chi^{2}(2$, $N=71)=7.82, p=0.02$.

In the introduction to this experiment, we gave two possible reasons for predicting more correct interpretations of DF than of MF equations. One reason was that people should be more likely to interpret correctly the equations that they understand as corresponding to problem solving 
Table 4 Modeling interpretations of multiplication format (MF) and division format (DF) equations in Experiment 3

\begin{tabular}{|c|c|c|}
\hline $\begin{array}{l}\text { Equation } \\
\text { format }\end{array}$ & Correctness & Illustrative modeling interpretations (equivalence or computation) \\
\hline \multirow[t]{3}{*}{$\operatorname{MF}(N=34)$} & Correct $(44 \%)$ & $\begin{array}{l}Y=9 X ; \mathrm{X}=\# \text { of cars during the day; } \mathrm{Y}=\# \text { of cars at rush hour "During rush hour, the number of } \\
\text { people driving is multiplied by } 9 . " \text { (Computation) }\end{array}$ \\
\hline & Reversal error (44\%) & $\begin{array}{l}9 X=Y ; \mathrm{X}=\# \text { of candy bars; } \mathrm{Y}=\# \text { of dollars "The value of nine candy bars is equivalent to the value } \\
\text { of one dollar." (Equivalence) }\end{array}$ \\
\hline & Other error $(12 \%)$ & $\begin{array}{l}X=6 Y ; \mathrm{X}=\# \text { of dollars; } \mathrm{Y}=\# \text { of cupcakes " } 6 \text { cupcakes }(\mathrm{Y}) \text { costs }(\mathrm{X}) \text { amount of dollars. This is a } \\
\text { relationship between cost/price and purchase/item." (Equivalence) }\end{array}$ \\
\hline \multirow[t]{3}{*}{$\mathrm{DF}(N=37)$} & Correct $(76 \%)$ & $\begin{array}{l}X / 7=Y ; \mathrm{X}=\# \text { of apples; } \mathrm{Y}=\# \text { of oranges "The number of apples divided by seven will give you the } \\
\text { number of oranges." (Computation) }\end{array}$ \\
\hline & Reversal error $(16 \%)$ & $X / 6=Y ; \mathrm{X}=\#$ of six packs; $\mathrm{Y}=\#$ of beer cans "There are six cans of beer in a six-pack." (Equivalence) \\
\hline & Other error $(8 \%)$ & $\begin{array}{l}X=Y / 6 ; \mathrm{X}=\# \text { of pizza slices; } \mathrm{Y}=\# \text { people "As the number of people increase, the number of pizza } \\
\text { slices decreases by a factor of } 6 . " \text { (Equivalence) }\end{array}$ \\
\hline
\end{tabular}

solutions (C), than equations that they view as algebraic models of equivalence relations (E). Indeed, collapsing across the MF and DF conditions, the proportion of correct C interpretations (89\%) was significantly higher than the proportion of correct $\mathrm{E}$ interpretations (43\%), $\chi^{2}(1, N=71)$ $=14.64, p<0.001$. Interestingly, there was a significant interaction between the type of modeling interpretation participant made and the format of the equation they interpreted, $\chi^{2}(3, N=71)=9.70, p=0.02$. Specifically, the proportion of correct $\mathrm{C}$ interpretations was similarly high in both format conditions $(89 \%$ and $88 \%$, for the DF and MF equations, respectively). By contrast, the proportion of correct $\mathrm{E}$ interpretations was significantly higher among participants who interpreted DF equations (61\%) than among those who interpreted MF equations (31\%). This interaction is consistent with the second reason we gave for predicting a higher proportion of correct interpretations in the DF than in the MF condition, namely, MF equations afford direct translation whereas DF equations do not.

The equivalence interpretations participants provided $(\mathrm{E})$ were similar to the verbal comparison statements we presented to the participants in Experiments 1 and 2 . Indeed, consistent with the results of the first two experiments, those participants who came up with E interpretations of MF equations were very likely to commit reversal errors. By contrast, those who came up with $\mathrm{C}$ interpretations of the MF and the DF equations did not commit such errors. Evidently, participants who made $\mathrm{C}$ interpretations were able to recognize the relevance of mathematical modeling, which they acquired in the context of solving numerical word problems, to the task of interpreting algebraic equations.

\section{Discussion}

The results of Experiment 3 show that mathematically equivalent algebraic equations, which differ only in their format, tend to evoke qualitatively different types of modeling interpretations. Standard-form equations (MF) were more likely than non-standard-form equations (DF) to evoke an interpretation that the algebraic equation represents an equivalence relation between two quantities. In a complementary way, DF equations were more likely than MF equations to evoke an interpretation that the algebraic equation represents problem-solving computations. These findings suggest that the exclusive use of the standard form, which students are encouraged to use when dealing with linear equations in a variety of algebraic modeling tasks (e.g., Larson, Kanold, \& Stiff, 1993; Rubenstein et al., 2002), leads a substantial proportion of college students to separate this representational meaning of algebraic models from the fact that these models also represent arithmetic computations, of the sort one would do in the context of solving numerical word problems.

The possibility that the format of algebraic models is understood to denote a conceptual distinction between representations of equivalence $(\mathrm{MF})$ and representations of arithmetic computations (DF) is consistent with the claims made by Sfard (1991) about students' difficulties with understanding that algebraic equations and arithmetic computations are akin to two sides of the same mathematical coin (see also Kieran, 1990, 1992). Such difficulties may be due to the fact that the concept of mathematical equivalence is not highlighted in arithmetic instruction. Indeed, many grade-school students believe that the equal sign indicates that they should calculate the answer of a given problem and do not understand that it denotes mathematical equivalence (e.g., Baroody \& Ginsburg, 1983; Cobb, 1987; McNeil \& Alibali, 2005). The results of Experiment 3 suggest that college students, who most likely understand the concept of mathematical equivalence, have learned to associate this concept with standard form algebraic models, but maintain that mathematically equivalent DF equations represent problem solving solutions. 
The fact that, for both equation formats, students' modeling interpretations were more likely to be correct when they made Computational than when they made Equivalence interpretations is consistent with our entering conjecture that people's ability to relate algebraic representations to their previously acquired knowledge of numerical problem solving reduces their reliance on the non-modeling direct translation method. However, students' tendency to come up with $\mathrm{E}$ or $\mathrm{C}$ interpretation did depend on the format of the algebraic equation. The standard form $\mathrm{MF}$ equations evoked a high proportion of $\mathrm{E}$ interpretations and led to a high proportion of reversal errors. By contrast, the non-standard DF equations evoked a higher proportion of $\mathrm{C}$ interpretations, which were likely to be correct. Thus, the conceptual distinction some students made between the two equation formats, $\mathrm{E}$ and $\mathrm{C}$, further contributed to the discrepancy in performance between $\mathrm{MF}$ equations that afford direct translation and DF equations that do not.

\section{General discussion}

Three decades of research document that a large proportion of college students commit reversal errors when they model verbal relational statements with algebraic equations. As we have described in the Introduction, such algebraic modeling errors have been explained by poor understanding of algebra and its relation to numerical problem solving. The studies reported here examined whether, and if so how, the standard multiplication form of algebraic models is responsible for the prevalence of reversal errors in college students. Using mathematically equivalent standard (multiplication) and non-standard (division) form algebraic equations, we found that most college students have sufficient understanding of algebraic modeling to construct correct algebraic models when using the non-standard format (Experiment 1), although this understanding may be supported by their modeling experience in the context of solving numerical word problems (Experiment 3). Furthermore, a significant majority of college students understand the mathematical equivalence between the standard and the non-standard algebraic models (Experiment 2). Thus, contrary to the common view, most reversal errors committed by college students do not reflect poor understanding of algebraic modeling.

Although the standard form appears to separate algebraic modeling from arithmetic modeling (Experiment 3), the main reason people who can construct correct algebraic models commit reversal errors seems to be that the surface similarity between the standard form of algebraic models and the linear form of verbal statements supports a one-to-one alignment between the words in the statement and the symbols in an algebraic equation. Exploiting this alignment, via the direct translation heuristic, is highly compelling and much faster than algebraic modeling. In fact, the results of the Experiment 2 show that many college students, who obviously can construct correct non-standard divisionformat models (Experiment 1), tend to select reversed DF models. As we have argued in the discussion section of Experiment 2, these students first translated verbal statements into (reversed) standard-form models and, subsequently, used their knowledge of mathematical equivalence to transform these standard-form models into (reversed) division-format models.

The fact that people who have good understanding of a domain nonetheless exploit surface cues that are correlated with the solution-relevant structure is consistent with prior work on "content effects" in the context of problem solving (e.g., Bassok et al., 1998; Blessing \& Ross, 1996; Novick, 1988). In our study, instead of content (e.g., objects, story line) the surface cue was the format of the algebraic equations. In some respects, college students' tendency to follow the linear layout of standard-form algebraic models (i.e., engage in direct translation of word to equations) is similar to recent findings of Landy and Goldstone (2007). These researchers found that the physical layout of algebraic equations affects the way college students apply a well-known mathematical rule. Specifically, they varied the spacing between arguments of addition and multiplication operations in algebraic equations and found that, when the spacing was consistent with the rule that multiplication precedes addition (e.g., " $g^{*} m+r^{*} w=m^{*} g+w^{*} r^{\prime}$ ), participants' judgments about the validity of such equations were faster and more accurate than when the spacing was inconsistent with this rule (e.g., " $s * n+e * c=n * s+c * e$ "). Of course, there are important differences between the tasks and the methodology in Landy and Goldstone (2007) experiments and in the experiments reported here. Nonetheless, both sets of studies show that the conventional physical form of notational tools affects people's algebraic performance.

In addition to the fluency with which people follow the layout of the standard form, which is disrupted by having to construct division-format models (Experiments 1 and 2), the results of Experiment 3 indicate that standard form equations (e.g., $7 X=Y$ ) are more likely than division-format equations (e.g., $X / 7=Y$ ) to elicit models of equivalence (e.g., "For every seven athletes on the team there is one coach."), whereas division-format equations are more likely than standard form equations to elicit models of problemsolving solutions (e.g., "The number of apples divided by 7 will give you the number of oranges."). As we have suggested in the discussion of Experiment 3, this difference in modeling interpretations may reflect students' selective experience with the modeling tasks they have encountered in algebra, whereby they have learned to associate standardform algebraic models solely with equivalence statements. 
As a result, the only association they have with divisionformat equations is with computations involved in numerical problem solving.

It is possible that, in addition to the effects of students' instructional history, the linear layout of a multiplicationformat equation lends itself better to a representation of equivalence relation than does the layout of a division-format equation, which highlights the asymmetry between the compared sets (Bassok et al., 1998). This possibility is consistent with the general argument, put forward by Landy and Goldstone (2007), that perceptual factors can affect algebraic reasoning. Although their experiments do not provide evidence that the perceptual layout of algebraic equations affects students' interpretation of the syntactic rule they had to apply, several studies suggest that the layout of the algebraic models people construct, or select, corresponds to their mental representations of the quantitative relations they attempt to represent (e.g., Mochon \& Sloman, 2004; Sherin, 2001; Waldmann, 2007). For example, Mochon and Sloman (2004) examined how people represent causal relations with algebraic equations. They found that people prefer algebraic models in which the causes are grouped on one side of the equal sign and the effect is on the other side to models that do not separate causes from effects.

Of course, more research is needed to examine how people's tendency to coordinate "percepts and concepts" (e.g., Goldstone \& Barsalou, 1998) interacts with their selective experience with the notational conventions they encounter in school. Such research is likely to have important implications for the design of instruction, be it in algebra or in other formal domains that require the use of representational tools (e.g., see Novick \& Catley, 2007, for similar arguments pertaining to the use of diagrammatic representations in biology). Whatever the outcomes of such future studies may be, the present studies show that the standard-form convention hinders people's performance on algebraic modeling tasks, even when they appear to have sufficient understanding of algebraic modeling, and fosters a conceptual separation between algebraic representations of equivalence and arithmetic computations that are involved in solving numerical word problems.

\section{References}

Baroody, A. J., \& Ginsberg, H. P. (1983). The effects of instruction on children's understanding of the "equals" sign. The Elementary School Journal, 84, 198-212.

Bassok, M., Chase, V., \& Martin, S. A. (1998). Adding apples and oranges: Alignment of semantic and formal knowledge. Cognitive Psychology, 35, 99-134. doi:10.1006/cogp.1998.0675.

Bassok, M., Wu, L. L., \& Olseth, K. L. (1995). Judging a book by its cover: Interpretative effects of content on problem-solving transfer. Memory \& Cognition, 23, 354-367.
Bernardo, A., \& Okagaki, L. (1994). Roles of symbolic knowledge and problem-information context in solving word problems. Journal of Educational Psychology, 86, 212-220. doi:10.1037/ 0022-0663.86.2.212.

Blessing, S. B., \& Ross, B. H. (1996). Content effects in problem categorization and problem solving. Journal of Experimental Psychology: Learning, Memory, and Cognition, 22, 792-810. doi:10.1037/0278-7393.22.3.792.

Borchert, K. (2003). Disassociation between arithmetic and algebraic knowledge in mathematical modeling. University of Washington: Unpublished Dissertation.

Clement, J. (1982). Algebra word problem solutions: Thought processes underlying a common misconception. Journal for Research in Mathematics Education, 13, 16-30.

Clement, J., Lochhead, J., \& Monk, G. S. (1981). Translation difficulties in learning mathematics. The American Mathematical Monthly, 88, 286-290.

Clement, J., Lochhead, J., \& Soloway, E. (1979). Translating between symbol systems: Isolating a common difficulty in solving algebra word problems. A cognitive development project, department of physics and astronomy. Amherst, Massachusetts: University of Massachusetts.

Cobb, P. (1987). An investigation of young children's academic arithmetic contexts. Educational Studies in Mathematics, 18, $109-124$

Cohen, E., \& Kanim, S. E. (2005). Factors influencing the algebra "reversal error. American Journal of Physics, 73, 1072-1078.

Dixon, J. A. (2005). Mathematical problem solving: the roles of exemplar, schema, and relational representations. In J. I. D. Campbell (Ed.), Handbook of mathematical cognition (pp. 379395). New York, NY: Psychology Press.

Dixon, J. A., Deets, J. K., \& Bangert, A. (2001). The representations of the arithmetic operations include functional relationships. Memory \& Cognition, 29, 462-477.

Fisher, K. M. (1988). The students-and-professors problem revisited. Journal of Research in Mathematics Education, 19, 260-262.

Goldstone, R. L., \& Barsalou, L. W. (1998). Reuniting perception and conception. Cognition, 65, 231-262. doi:10.1016/S0010-0277 (97)00047-4.

Graf, E. A., Bassok, M., Hunt, E., \& Minstrell, J. (2004). A computer-based tutorial for algebraic representation: The effects of scaffolding on performance during the tutorial and on a transfer task. Technology Instruction Cognition and Learning, 2, 135-170.

Herscovics, N. (1989). Cognitive obstacles encountered in the learning of algebra. In S. Wagner \& C. Kieran (Eds.), Research issues in the learning and teaching of algebra (pp. 60-86). Hillsdale, NJ: Erlbaum.

Herscovics, N., \& Linchevski, L. (1994). A cognitive gap between arithmetic and algebra. Educational Studies in Mathematics, 27, 59-78. doi:10.1007/BF01284528.

Hinsley, D. A., Hayes, J. R., \& Simon, H. A. (1977). From words to equations: Meaning and representation in algebra word problems. In M. A. Just \& P. A. Carpenter (Eds.), Cognitive processes in comprehension (pp. 89-106). Hillsdale, NJ: Erlbaum.

Kaput, J., \& Sims-Knight, J. E. (1983). Errors in translations to algebraic equations: Roots and implications. Focus on Learning Problems in Mathematics, 5, 63-78.

Kieran, C. (1990). Cognitive processes involved in learning school algebra. In P. Nesher, J. Kilpatrick, et al. (Eds.), Mathematics and cognition: A research synthesis by the international group for the psychology of mathematics education (pp. 97-136). Cambridge, England: Cambridge University Press.

Kieran, C. (1992). The learning and teaching of school algebra. In D. A. Grouws (Ed.), Handbook of research on mathematics teaching and learning (pp. 390-419). New York: Macmillan. 
Knuth, E. J., Alibali, M. W., McNeil, N. M., Weinberg, A., \& Stevens, A. C. (2005). Middle school students' understanding of core algebraic concepts: Equivalence \& variable. International Journal on Mathematics Education, 37, 68-76. doi:10.1007/BF02655899.

Koedinger, K. R., Alibali, M. W., \& Nathan, M. J. (2008). Trade-offs between grounded and abstract representations: Evidence from algebra problem solving. Cognitive Science, 32, 366-397. doi:10.1080/03640210701863933.

Kuchemann, D. (1981). Algebra. In K. Hart (Ed.), Children's understanding of mathematics: 11-16 (pp. 102-119). London: Murray.

Landy, D., \& Goldstone, R. L. (2007). How abstract is symbolic thought? Journal of Experimental Psychology: Learning, Memory, \& Cognition, 33, 720-733. doi:10.1037/0278-7393.33.4.720.

Larson, R. E., Kanold, T. D., \& Stiff, L. (1993). Algebra 1. Lexington, MA: D. C. Heath and Company.

Lochhead, J., \& Mestre, J. P. (1988). From words to algebra: Mending misconceptions. In A. Cosford \& A. Schulte (Eds.), The idea of algebra k-12: National Council of Teachers of Mathematics Yearbook (pp. 127-136). Reston, VA: National Council of Teachers of Mathematics.

MacGregor, M. E., \& Stacey, K. (1993). Cognitive models underlying students' formulation of simple linear equations. Journal for Research in Mathematics Education, 24, 217-232. doi:10.2307/749345.

Martin, S. A., \& Bassok, M. (2005). Effects of semantic cues on mathematical modeling: Evidence from word-problem solving and equation construction tasks. Memory \& Cognition, 33, 471478.

Mayer, R. E., \& Hegarty, M. (1996). The process of understanding mathematical problems. In R. J. Sternberg et al. (Eds.), The nature of mathematical thinking: The studies in mathematical thinking and learning series (pp. 29-53). Mahwah, N.J.: Erlbaum.

McNeil, N. M., \& Alibali, M. W. (2000). Learning mathematics from procedural instruction: Externally imposed goals influence what is learned. Journal of Educational Psychology, 92, 734-744. doi:10.1037/0022-0663.92.4.734.

McNeil, N. M., \& Alibali, M. W. (2005). Knowledge change as a function of mathematics experience: All contexts are not created equal. Journal of Cognition and Development, 6, 285-306. doi:10.1207/s15327647jcd0602 6 .

Mochon, D., \& Sloman, S. A. (2004). Causal models frame interpretations of mathematical equations. Psychonomic Bulletin \& Review, 11, 1099-1104.

Novick, L. R. (1988). Analogical transfer, problem similarity, and expertise. Journal of Experimental Psychology: Learning, Memory, and Cognition, 14, 510-520. doi:10.1037/0278-7393.14.3.510.

Novick, L. R., \& Catley, K. M. (2007). Understanding phylogenies in biology: The influence of a Gestalt perceptual principle. Journal of Experimental Psychology: Applied, 13, 197-223. doi:10.1037/ 1076-898X.13.4.197.

Rosnick, P. (1981). Some misconceptions concerning the concept of variable. Mathematics Teacher, 74, 418-420.

Rosnick, P., \& Clement, J. (1980). Learning without understanding: The effect of tutoring strategies on algebra misconceptions. Journal of Mathematical Behavior, 3, 3-27.

Rubenstein, R. N., Craine, T. V., Butts, T. R., Cantrell, K., Dritsas, L., Elswick, V. A., et al. (2002). Integrated mathematics 1, teacher's edition. McDougal Littell: Evanston, IL.

Sfard, A. (1991). On the dual nature of mathematical conceptions: Reflections on processes and objects as different sides of the same coin. Educational Studies in Mathematics, 22, 1-36. doi:10.1007/BF00302715.

Sherin, B. L. (2001). How students understand physics equations. Cognition and Instruction, 19, 479-541. doi:10.1207/S1532690XCI1904_3.

Smith, J. E. K. (1976). Data transformations in analysis of variance. Journal of Verbal Learning and Verbal Behavior, 15, 339-346. doi:10.1016/0022-5371(76)90030-X.

Stacey, K., \& MacGregor, M. (1999). Learning the algebraic method of solving problems. Journal of Mathematical Behavior, 18, 149-167. doi:10.1016/S0732-3123(99)00026-7.

Waldmann, M. R. (2007). Combining versus analyzing multiple causes: How domain assumptions and task context affect integration rules. Cognitive Science, 31, 233-256.

Wollman, W. (1983). Determining the sources of error in a translation from sentence to equation. Journal for Research in Mathematics Education, 14, 169-181. 